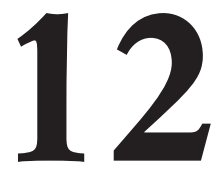

\title{
PEDAGOQUEER: RESISTENCIAS Y SUBVERSIONES EDUCATIVAS
}

\section{(QUEER PEDAGOGY: EDUCATIVE RESISTANCE AND SUBVERSIONS)}

\author{
Jordi Planella y Asun Pie \\ Universitat Oberta de Catalunya
}

$Y$ me pregunto si, al mantener esta tensión, la pedagogía queer podrá hacer que todos los implicados en la misma tengan en cuenta las condiciones de sus propias posibilidades, de sus propias inteligibilidades, además de su labor proliferadora de identificaciones y críticas propias que puedan superar el concepto de identidad como esencia, explicación, causalidad o trascendencia.

Britzman [2002, 201]

\section{RESUMEN}

En el presente texto se revisan las aportaciones de la teoría queer en los discursos y prácticas pedagógicas. El análisis se centra en las temáticas de desestabilización de la heternormatividad y la normalidad, los discursos sobre el cuerpo y las paradojas del debilitamiento de las identidades políticas. Por otro lado, se abren algunos interrogantes sobre la posibilidad/imposibilidad de la pedagogía para alejarse de juicios normalizadores, apuntando a una despedagogización de las prácticas o a una apertura de la misma idea de lo que es la educación, especialmente en su articulación con la experiencia y el acontecimiento. Finalmente se apunta la posibilidad de aplicación de la teoría queer al ámbito de la discapacidad y al terreno de la radical diferencia corporal. El cuerpo se presenta aquí como central para la subversión y resistencia de la misma idea de normalidad. Específicamente, dicha resistencia se encarna en la recuperación de la dimensión simbólica del cuerpo.

\section{ABSTRACT}

In the present text the contributions of the queer theory in the speeches and pedagogical practices are reviewed. The analysis is centred in theme of destabilization of the heteronormativity and normality, the speeches on the body and the paradoxes of the weakening of the political identities. On the other hand, these issues bring some questions on the possibility/impossibility of pedagogy to move away from normalizations judgments, aiming at a desedu- 
cation of the practices or an opening of the same idea of which it is the education, specially in its joint with the experience and the event. Finally the analysis points to the possibility of the application of the queer theory to the dimension of the disability and the field of the radical corporal difference. The body appears here like the power station for the subversion and resistance of the same idea of normality. Specifically, this resistance is incarnated in the recovery of the symbolic dimension of the body.

\section{SITUANDO LA TEORÍA QUEER}

Lo queer ha llegado y atraviesa nuestras vidas, nuestras instituciones, nuestras relaciones y formas de mirar y encuadrar el mundo. Hasta hace poco las agencias educativas se mantenían encerradas en sí mismas, como formas de anquilosamiento institucional, como garante social de las formas de la normalidad, y como actor de los medios de control de las subjetividades excesivamente perturbadoras. Pero ese resistirse a abrir los ojos ha llevado a un cierto desbordamiento de las formas y los estilos de acoger y recoger nuevos discursos y nuevas prácticas pedagógicas. En el presente artículo nos hacemos eco y revisamos la introducción de la teoría queer en los discursos y prácticas pedagógicas, y nos posicionamos como forma emergente de pedagogía de la resistencia y de la subversión.

Las formas que la pedagogía tiene de construir las normatividades corporales, en lo que se refiere a la sexualidad, ha llevado a pedagogos/as, maestros y educadores/as a repensar qué hacen, cómo lo hacen y qué producen en sus praxis en relación con las temáticas sexuales. Es cierto que la publicación del trabajo de Butler Gender Trouble sirvió de punto de partida para reflexionar y pensar de otra forma la pedagogía del género y de la sexualidad. Pero no se trata únicamente de la influencia del trabajo de Butler, sino que la Queer Theory puede ser considerada la respuesta a dos hechos específicos, por lo menos en lo que se refiere al desarrollo en los EEUU. Por una parte, es la respuesta a la creciente tendencia neoconservadurista norteamericana, que fue liderada por la Sentencia del Tribunal Supremo de los EEUU (caso Bowers contra Hardwick, 1986) de condenar las prácticas «sodomitas» en el Estado de Georgia (Peter, 2000) [1]. Y por otra, es el resultado de la organización social frente a la creciente expansión de la epidemia del sida.

Posiblemente sea necesario plantearse algunas preguntas antes de seguir abordando el por qué del olvido, consciente o inconsciente, de estas temáticas. Algunas de las cuestiones son: ¿Quién se ha dedicado a la producción de teoría en los campos sexuales no normativos? ¿Los investigadores de la teoría GLBT (Gays, Lesbianas, Bisexuales y Transexuales) son ac- 
tivistas de estos movimientos o simplemente investigadores interesados en temas fronterizos y transgresores o contestatarios?

Una posible respuesta se encuentra en el hecho de que las estructuras universitarias, son territorios hiperregulados, y, de forma «invisible», existen determinadas formas de censura en la producción de conocimiento y en la selección de los temas de interés para las investigaciones. A esta causa, más centrada en aspectos institucionales, se le puede añadir la que propone Bourcier: «algunos profesores, próximos a los intelectuales mediáticos, permanecen sordos y ciegos a los puntos de vista de estudiantes, de minorías, de sus subalternos, ejerciendo un poder de inercia en el cual la persona ignora las capacidades» (2002). Pero escuchar a los demás y plantearse nuevos objetos de estudio, otros temas de interés, nuevas epistemologías y hacerlo con la intención de desestabilizar determinadas construcciones binarias de la realidad, comporta el peligro de que la academia considere al investigador, al pedagogo y/o al profesor como pensador naif, en contraposición con la adultez de aquellos que se centran en temas más formales.

\section{LA CUESTIÓN QUEER EN LA PEDAGOGÍA}

Existen múltiples conexiones entre la teoría queer y la pedagogía que vienen dadas por algunas personas que forman parte de grupos GLBT o bien que se sienten atraídos por sus discursos y formas de entender las relaciones sociales. Fundamentalmente se trata de personas que buscan posiciones más allá de la heteronormatividad y la normalidad como elementos de estabilidad pedagógica. Si revisamos la producción sobre la temática queer en nuestro país, nos daremos cuenta que prácticamente no existen trabajos, menos aún si los buscamos ligados directamente a aspectos pedagógicos de la teoría. Es seguramente una excepción la publicación del capítulo de Britzman «La pedagogía transgresora y sus extrañas técnicas», dentro de la obra coordinada por Mérida Sexualidades transgresoras. Una antología de estudios queer (2002). En la presentación del volumen, Mérida resume la propuesta de Britzman diciendo que: «el aula puede transformarse en un espacio que favorezca el cambio social si la práctica docente conjuga una revisión de la estructura autoritaria que suele definir sus estrategias y, sobre todo, con el cuestionamiento cotidiano de la heterosexualidad normativa a través del modelo de aprendizaje trasgresor» $(2002,25)$.

El punto de partida de este encuentro entre lo queer y la pedagogía podría situarse en una reflexión que Spivak hace: debemos pensar sobre la forma en que la educación institucional o conjunto de discursos y prácticas se encuentra relacionada con la autodeterminación de las poblaciones su- 
balternas del mundo, así como de su subordinación (Spivak, 1992). Esta reflexión se ve apoyada por los interrogantes que plantea Britzman: «¿Es posible que el proyecto educativo se convierta algún día en un punto de encuentro para las revueltas deconstructivas? ¿Podría la pedagogía suscitar reacciones éticas que fueran capaces de rechazar las condiciones normalizantes del origen y del fundamentalismo, aquellas que rechazan la sumisión?»(Britzman, 2002, 197).

De esta forma los trabajos sobre la teoría queer empiezan a entrar con una cierta facilidad en el terreno pedagógico, especialmente en el contexto geográfico de Australia, Canadá y los EE.UU. Trabajos como los de Britzman (2002), Gough y Gough (2002), McKeehen (2002), Grace (2001), Kopelson (2002), Pinar (1998), Curran (2002), Woog (1995), Weems (1999) y Unks (1995) son claros ejemplos de este nuevo pensamiento hermenéutico y epistemológico. Una parte de los trabajos se elaboran desde la teoría de la educación y la teoría del currículum, y algunos se publican en revistas y monografías de la correspondiente área de conocimiento. Este es el caso de Britzman que publica su primer trabajo sobre pedagogía queer en 1995 en Educational Theory en el cual abre el terreno para asentar las bases de esta nueva perspectiva pedagógica. Britzman habla abiertamente de su influencia en la construcción de su modelo pedagógico basado en la recepción de ideas de Freud, Foucault y Butler:

Estos tres discursos, juntamente con toda la teoría y la experiencia de los movimientos y discursos GLBT, posibilitan partir de una nueva epistemología que no obliga a pensar en criterios binómicos. La epistemología queer desestabiliza las identidades sexuales, situando de lado la homosociabilidad y la homosexualidad (Sedgwick, 1998) y ofreciendo alternativas para pensar, estructurar y normativizar las praxis pedagógicas.

\section{DECONSTRUIR PEDAGÓGICAMENTE LA ANORMALIDAD CORPORAL}

Hablar de pedagogía queer lleva inherente la referencia al término normal/anormal o normalidad/anormalidad. A través de un ejercicio sutil, aquellos sujetos que no encajan en la definición de normal son enviados a la nueva categoría de a-normales. No encontramos únicamente sujetos con determinadas tendencias sexuales, sino que cada vez más encontraremos un conjunto importante de sujetos que «escapan» a la definición de normales, debido a múltiples factores. De esta binarización de la realidad (pasada por el filtro de los imaginarios) fácilmente se desprende que la categoría heterosexual se complementa con los adjetivos bueno, normal y natural, mientras 
que la categoría homosexual se complementa con los adjetivos malo, anormal y desnaturalizado. A los primeros les corresponden beneficios y un estatus reconocido, mientras que para los segundos quedan los estigmas, los roles desvalorizados y los intentos de regulación social. En esta línea de la construcción social de la problematicidad, Curran sugiere que es importante contestar la evidencia natural de las categorías identitarias y poder desnaturalizar los relatos binarios existentes en clave hombre/mujer y homo/hetero (Curran, 2002). El trabajo de campo de Curran sobre la forma como los profesores conciben y transmiten las corporeidades ligadas a los temas queer le permite ofrecer esta amplia perspectiva en torno al tema que nos ocupa. Estudia también la situación de los jóvenes queers en las instituciones educativas de secundaria de Melbourne y llega a las siguientes conclusiones:

- constituyen un grupo de riesgo con una serie de necesidades especiales

- la homosexualidad es percibida como una amenaza para los compañeros

- las actuaciones queer de los profesores pueden servir de ejemplo a los estudiantes queer (Curran, 2002)

Si bien el trabajo de Curran es ilustrativo de lo que se produce bajo la denominación de pedagogía queer, no es la única línea de pensamiento existente, ya que el objetivo de la pedagogía queer no se limita ni se centra exclusivamente en los temas ligados con la vivencia de las identidades GLBT. Lo que busca la pedagogía queer es la desestabilización del binomio normal/anormal. Lo afirma Britzman con claridad:

Todas estas prácticas despiertan nuestra curiosidad sobre el modo en que la normalidad se convierte en un elemento enormemente imperceptible en el aula, y sobre cómo la propia pedagogía puede intervenir para hacer perceptibles los límites y los obstáculos de la normalidad (Britzman, 2002, 199).

Desde la posición construccionista, la pedagogía queer no admite la definición de personas o situaciones «normales» a las cuales el resto deberían de poder aspirar a imitar. En este sentido los trabajos de Britzman (2002) girarán en torno al concepto de normalidad y a las teorías y praxis que pueden deconstruirlo. La teoría queer, el psicoanálisis y diferentes pedagogías proponen una pedagogía «transgresora». Se quiere romper con la idea de otro como sujeto sospechoso, peligroso, temeroso, infeccioso, preocupante y amenazante (para el resto). En la pedagogía queer no existe un «manual de instrucciones pedagógicas». Es necesario fundamentar toda esta tarea en la misma hermenéutica, en la interpretación de la discursividad; una discursi- 
vidad que a través del lenguaje construye y deconstruye la línea que separa la normalidad de la anormalidad.

La posición binaria entre lo normal y lo anormal necesita ser deconstruida, aunque no a través de la reconducción de los sujetos ubicados en la categoría «anormal», sino a través de lo que propone Wiegman: «explorar un nuevo imaginario político en el que puedan forjarse diversas alianzas entre las personas que no se reproducen, entre los excéntricos del género, los bisexuales, los gays y las lesbianas, los no monógamos-, alianzas que pueden empezar e innovar las formas de disciplina social e intelectual de la academia» $(2002,177)$.

\section{LAS TRAMPAS DE LA IDENTIDAD}

La teoría queer se aleja de la victimización y la normalización. Como ya se ha dicho, a pesar de que parezca una paradoja, no preocupa tanto el logro de unos derechos, en definitiva, heteronormativos, como la eliminación de aquellos discursos que mantienen y reproducen relaciones de dominación. El deseo de normalidad reniega de la misma particularidad subjetiva y refuerza una posición sumisa. La destrucción de algunas categorías, fundamentalmente la de identidad, y con ella todas las prácticas que le son propias (asociacionismo, grupos identitarios, etc...) comporta una serie de riesgos que deben considerarse. El planteamiento queer distorsiona de tal manera el concepto de identidad que pone en peligro la lucha política del movimiento gay y lésbico. Se cuestiona la misma idea de colectivo homosexual debilitando así la lucha y militancia política basada en las identidades sexuales. Se pone en cuestión la misma utilidad política de las identidades sexuales.

Bajo la mirada queer, las identidades quedan completamente desarticuladas, son fluidas y cambiantes; se convierten en no-identidades. Las identidades provocadoras trabajan para interrumpir los discursos que limitan a los seres humanos. Lo queer es aquello que contesta la normalización pedagógica y encuentra maneras para apropiarse de los cuerpos. Convertir dichas apropiaciones en categorías identitarias estables es algo que dista mucho de la dinámica queer y que, más bien, está relacionado con estructuras de dominación que intentan reformular lo queer para someterlo a la lógica imperante.

Las identidades son una ficción, a pesar de que tienen una utilidad: corporeizar una lucha política con el objetivo de lograr unos derechos legítimos. Pero esta lógica activista debe enfrentarse con uno de los objetivos 
centrales de la teoría queer: disolver las categorías de identidad. Se sostiene que las identidades sexuales son productos históricos y sociales y que la finalidad de fijarlas está relacionada, fundamentalmente, con el control social. El mismo binomio hombre/mujer excluye otras posibilidades y niega, en definitiva, el carácter constructivo del sexo y la sexualidad. El mundo, y con él, las instituciones, se estructura según esta lógica. Así, las reglamentaciones, las definiciones, las organizaciones sociales parten de este supuesto binario. Como nos recuerdan Berlant y Warner «La heterosexualidad implica tal cantidad de prácticas que no son sexuales que, en este momento, es inimaginable un mundo donde este compendio de normas hegemónicas no sea dominante» (Berlant y Warner 2002, 242). La posición queer cuestiona la mayor parte de los recursos educativos y sociales que se imponen bajo esta lógica heterosexista. Así pues, ¿ es posible la misma idea de institución y de educación desde los parámetros queer? En nuestra realidad, se trata de ser algo, pero ser algo o alguien que se ajuste a las categorías establecidas, significa estar dentro o estar fuera. La política queer no solo se opone a la idea de normalidad sino a la misma idea de comportamiento normal. Los sujetos queer no desean ser normales, no desean estar dentro. Como dice Gamson, la dinámica de «o bien esto, o bien esto otro», o «eres gay o heterosexual» es una cuestión más que está relacionada con el carácter heternormativo de las instituciones. Los gays no existen, del mismo modo que tampoco existen los heterosexuales. Las identidades son cambiantes y fluidas, las categorías son sociales e históricas. Bornstein nos recuerda: "¿Acaso distinguimos a un hombre de una mujer por su anatomía?» (Bornstein citado por Gamson 2002, 158). La aceptación de la homosexualidad no es el tema que nos ocupa, ni interesa al discurso queer, sino la deconstrucción del código hetero/homo. Así pues, el hecho de que las instituciones toleren y respeten la homosexualidad no es trascendente. Y no lo es porque, como hemos visto, se continúa manteniendo la misma lógica cerrada. La sexualidad humana es demasiado compleja para encerrarla en dos palabras limitadas. La mayor parte de su complejidad está relacionada con la imposibilidad de nombrarla, como mínimo, en su totalidad. El rechazo o aceptación de la homosexualidad, decíamos, no es el tema que nos ocupa, pero la cuestión central y trascendental será preguntarse ¿por qué esta elección de objeto despierta tantas ansiedades? «(...) No se trata de por qué algunas personas lo encuentran atractivo, sino por qué tantas personas lo encuentran aborrecible». (Harris, citado por Lizarraga 2003, 197). Es posible que encontremos una respuesta en lo que Derrida llama alteridad absoluta. "El ser y los otros son vistos como totalmente externos, totalmente separados, totalmente otros. Y cuando los sujetos se posicionan en el exterior absoluto nacen las fobias». (Morris 2005, 46). 


\section{EL CUERPO: ELEMENTO CENTRAL DE LA TEORÍA QUEER}

En la pedagogía queer el cuerpo se ha convertido en un tema central y entendemos que es necesario que nos preguntemos: ¿qué ha llevado a una pedagogía de este tipo a fundamentar su discurso en la corporeidad? Una primera respuesta la encontramos en la reflexión de Llamas:

Tales procesos de subjetividad, de control de la propia vida, de determinación del propio destino, no pueden sino partir del cuerpo (...) Siendo sólo cuerpos, estamos (paradójicamente) en una posición privilegiada para conocernos, desarrollarnos, realizarnos e innovarnos, sin renunciar al placer ni a ninguno de los criterios de subjetividad metafísica. Sólo siendo cuerpos seremos algo más (Llamas, 1998).

El cuerpo, tal y como hemos descifrado, ha sido objeto de sumisión, imposición, docilización, anulación, negación, vejación, ignorancia, politización, pedagogización, etc. Parece lógico, pues, partir de una pedagogía que entienda esta posición del cuerpo en la subjetivización y que tenga presente la experiencia corporal de los sujetos. Los cuerpos de los sujetos «anormalizados» pueden convertirse fácilmente en «carne de cañón» para el resto de la sociedad. Sin la existencia del cuerpo negado no hay posibilidad de cuerpo aceptado y cuerpo vivido. Es en este sentido que se apuntan diferentes discursos pedagógicos.

\subsection{El conocimiento encarnado}

Una primera línea de discursos parte de los pedagogos/as, maestros, educadores/as que han vivido en sus propios cuerpos situaciones de opresión. Su propia experiencia es la primera fuente de conocimiento que les permitirá arrancar la producción de discurso queer. Un grupo significativo forma parte de colectivos GLBT, tal y como se desprende de Jennings (1994) cuando afirma que aproximadamente un 10\% del profesorado estudiado (Boston) son gays o lesbianas, y el trabajo de Khayatt (1992) sobre la presencia invisible de los profesores homosexuales en las aulas de los centros educativos de los EEUU. Pero no solamente se trata de educadores ligados a posiciones GLBT, sino de personas que pueden haber sufrido opresiones por su color de piel, por el hecho de ser mujer, por hablar otra lengua, por pensar diferente, por tener una discapacidad, etc. Una parte de los pedagogos queer son personas «diferentes» que ejercen abiertamente esta diferencia. Un ejemplo clarificador de lo que afirman es la narración de Sedgwick (profesora de la literatura inglesa en of New York) en relación con su experiencia de diagnóstico de cáncer de mama: 
En la época en que recibí el diagnóstico, los acontecimientos más inmediatos de mi vida eran, en primer lugar, una clase de doctorado sobre teoría queer, cuya docencia incluía materiales relacionados con el sida (Sedgwick, 2002, 45).

La autora parte de su propia experiencia de sufrimiento en su cuerpo (al margen de estar integrada en grupos GLBT). El trabajo para romper «pedagógicamente» las construcciones binarias se vive de forma diferente, porque algunos sujetos son educadores sociales que trabajan para eliminar situaciones que oprimen a los sujetos, pero a su vez sufren alguna situación de opresión/exclusión, por no ser heteronormativos, tener una discapacidad, provenir de una comunidad de origen diferente a la de acogida, etc. Otro ejemplo de esta situación particular de exclusión la encontramos en Audre Lorde. En cierto sentido Lorde terminó politizando e instrumentalizando su experiencia radical como negra y lesbiana apelando al retorno de esa parte femenina desterrada, olvidada. Aparecía, por lo tanto, una búsqueda de pacificación con ese interior no normativo, silenciado por las estructuras de poder. En síntesis, pensar cómo vivimos las situaciones de opresión en nuestros cuerpos nos puede ayudar a extrapolarlas a los discursos pedagógicos, convirtiéndose así, la experiencia, en una verdadera fuente de producción de conocimiento pedagógico. En un conocimiento encarnado.

\subsection{La hipercorporalización}

Una segunda línea parte de la hipótesis que los sujetos anormalizados han pasado a ser sujetos hipercorporalizados. El cuerpo ha tomado una relevancia desenfrenada y el sujeto ha sido "cosificado» y ahora es, sobre todo un cuerpo. Tal y como nos hace ver Hénaff: «el suplicio libertino hace avanzar y lleva al extremo la lógica de la reducción anatómico/quirúrgica del cuerpo, postulada por la ciencia» (Hénaff, 1980, 29). Es a través de los diferentes saberes (entre los cuáles se encuentra la pedagogía) que el sujeto pasa a ser solamente un cuerpo, quedando anulada su personalidad, su historia y su identidad. En dicha situación se encuentran los cuerpos intersexuales, algunos de los cuales han sufrido en sus carnes la agresión y la violencia de la normalización. La intersexualidad pone en tela de juicio la situación binaria de hombre/mujer y representa el paradigma de la construcción del género. La matriz heteronormativa, con sus dispositivos de poder médicos, es la encargada de restaurar y mantener dichos criterios de normalidad. A través de drásticos medios quirúrgicos, se asigna un sexo. «El hecho de que este sistema que preserva las fronteras de las categorías de varón o de mujer haya existido durante tanto tiempo sin despertar críticas ni escrutinio desde ningún flanco indica la incomodidad extrema que despier- 
ta la ambigüedad sexual en nuestra cultura». (Chase 2005, 67). La intersexualidad cortocircuita los sistemas heteronormativos de sexo, género y sexualidad generando una incomodidad más que destacable en los sujetos inscritos como normativos. La intersexualidad se concibe como una situación de desorden, con carácter de falsa o anómala sexualidad. Si el otro ha sido reducido a un cuerpo físico, debemos plantearnos la manera de contestar a los modelos de producción biopolítica. Y esta manera la encontramos, justamente, en la recuperación de la dimensión simbólica del cuerpo.

\subsubsection{El cuerpo resistente}

La tercera línea entiende el cuerpo como espacio/territorio de resistencia subjetiva. El cuerpo presenta esta doble perspectiva: espacio de imposición del poder y espacio de resistencia. Desde los colectivos GLBT hasta muchos otros grupos que proponen la recuperación de los cuerpos como espacios de inscripción subjetiva se reclama la necesidad de hablar de resistencia. Para McLaren «es importante comprender la resistencia a las modalidades dominantes de subjetividad, de producción y del deseo, especialmente si dicha resistencia está conectada somáticamente con la formación de la voluntad y con la construcción del significado» (1997, 96). La opción pasa por entendernos como productores de subjetividad, y esta acción requiere necesariamente volver a pensar y revisar el espacio de nuestros cuerpos en los espacios sociales. El cuerpo es entendido como espacio de lucha (nos marcamos corporalmente para ser diferentes del resto), de conflicto (los otros no siempre aprecian nuestros cuerpos, y esto les provoca múltiples reacciones) y de contradicciones (no estamos constituidos por cuerpos monolíticos, sino que pueden ser interpretados desde múltiples perspectivas). Para McLaren, la tarea de la pedagogía en relación con la resistencia corporal es la de «aumentar nuestra autoconciencia, de apartar la distorsión, de descubrir formas de subjetividad que sean consecuentes con el cuerpo/sujeto capitalista y que asistan al sujeto en su rehacer histórico» $(1997,97)$. Los cuerpos que resisten buscan significados más allá de las políticas de «normalización» corporal, y lo hacen rompiendo las estructuras y los discursos binarios. Para McLaren «la pedagogía del cuerpo/sujeto posmoderno puede ayudar a los educadores a comprender mejor cómo el cuerpo/sujeto resistente intenta significar más allá de la normativa y de los sistemas de significación disponibles, retando y rompiendo los discursos que crean el espacio de subjetividad (...) Para defenderse del miedo a la incertidumbre, del horror de la ambigüedad, y de la amenaza de la diferencia, los cuerpos/sujeto necesitan construir un lenguaje que rechace sus propios límites, que sea capaz de localizar los fallos y las fisuras de la hegemonía cultural que prevalece» $(1997,101-102)$. 
En este sentido es destacable el trabajo de Grace (2001) sobre teoría queer, los cuerpos y la educación de adultos. Grace propone una pedagogía que no excluya a los adultos de una formación en temas queer, ni tampoco excluya la posibilidad de que los adultos que asisten a instituciones educativas tengan identidades queer. Se trata de repensar la biografía del sujeto, encarnando las posibilidades que la narratividad ofrece a los sujetos adultos. Esta nueva perspectiva de afrontar la pedagogía queer desde la educación de adultos posibilita el aprendizaje corporal en la dimensión del Learning for Life. Podemos aprender a vivir en nuestro cuerpo a lo largo de la vida, a través de todos los cambios que experimenta, pero también podemos aprender a lo largo de la vida cosas sobre todos los otros cuerpos, que también cambian y se transforman a través del tiempo.

\section{LAS RAREZAS DE LA PEDAGOGÍA QUEER}

Los planteamientos queer son posiciones que van más allá de la heteronormatividad y la normalidad como elementos de estabilidad pedagógica. Esta presunta estabilidad está constituida por declaraciones reguladoras que no son objeto de reflexión. En este sentido, la educación mantiene cierta atracción hacia la verdad pura y las identidades estables ignorando, recurrentemente, las contradicciones. Dibujar una pedagogía «transgresora», preocupada por las exclusiones de la normalidad, permite la construcción de zonas alternativas de identificación y crítica necesarias para reconocer las estructuras dominantes y para la creación de nuevos deseos (Britzman 2002, 200).

El juicio normalizador es una constante en pedagogía. Se da, a menudo, a través de comparaciones de manera que se citan las acciones individuales con una referencia que debemos seguir (Gore 2000, 234). La referencia a las normas es una característica común de la pedagogía. Se invita a los sujetos a seguir una determinada norma, se citan, se anuncian, etc. Así, un marco normativo de referencia, el todo, espacio de diferenciación y principio de una regla es el heterosexismo. Educar, en sus términos más clásicos y prácticos ha consistido, entre otros, en enseñar normas de comportamiento, de conocimiento, actitudes. Aquí la productividad del poder parece un precepto fundamental de la actividad pedagógica (Gore 2000, 234).

La exclusión representa el lado oscuro de la normalización, la definición de lo patológico. La exclusión define la diferencia, las fronteras y las zonas. Las técnicas de exclusión son constantes en pedagogía; se excluyen individuos, identidades, prácticas, así como formas de construir el conocimiento. Por lo tanto, el interrogante esencial es ¿Qué referencia debe 
guiar a la pedagogía? Resulta casi impensable una pedagogía que no establezca límites, que no normalice y patologice. Pero al salir de estos discursos perversos nos encontramos con la posibilidad de dar todo el crédito a las autorepresentaciones de diferencia sexual ya que negar o alienar la autoridad de cualquier persona para describir o nombrar su propio deseo sexual es un acto de terribles consecuencias (Sedgwick 1998,40). "¿Puede, la pedagogía, reconocer la imposibilidad de pensar en la normalidad y la manera en que la normalidad se constituye una y otra vez?» (Britzman 2002, 212) Parece existir una limitación de las prácticas pedagógicas tecnocráticas o críticas para superar la exclusión en sus discursos. ¿La tarea de educar limita las mismas posibilidades de intervención? O bien ¿se trata de re-definir, reconfigurar la misma noción de educar? Como se ha comentado anteriormente, la fundamentación en la misma hermenéutica, en la interpretación de la discursividad, permite construir una pedagogía que supera las nociones de normalización, exclusión y clasificación. En definitiva, ¿puede pensarse una educación que supere las exclusiones producidas por una lógica metafísica? ¿puede pensarse una educación que sea contingente, contextual, perspectivista? ¿una pedagogía sin absolutos, sin Verdades? ¿Una pedagogía que no contenga certezas sino orientaciones provisionales? Para Mèlich educar no está relacionado con la transmisión del sentido de la vida porque el sentido no puede darse, sino que está relacionado con la formación de una razón imaginativa, capaz de narrar sentidos, en plural, porque nunca existe un único sentido sino sentidos diferentes (Mèlich 2010, 25). En esta lógica, nos acercamos al concepto de experiencia como acontecimiento en el que «algo» le sucede al sujeto. «Algo» acontece. Se trata de una especie de apertura de lo que es la educación. Experimentarse y experimentar la otredad propia y ajena son aquí centrales. Dejar de hablar de la otredad para sentirla y hacerlo des de la piel, la sensibilidad, desde los otros lenguajes que no capturan y reducen al otro con el propósito de hacerlo inteligible. El respeto hacia la alteridad no entiende de reducciones. La auténtica compasión -en términos de Mèlich- se ejerce desde la diferencia porque algunos cuerpos son, efectivamente, diferentes. Probablemente aquí la educación ya no es normalización, ni exclusión, ni clasificación. Es experiencia viva, encarnada, un registro hermenéutico que sitúa algunas prácticas educativas también en el escenario de la performatividad, de la puesta en escena de aquello que se pone en juego en la construcción del otro, del cuerpo-otro. Una narratividad puesta en escena. Vamos, pues, de las imposibilidades de la educación a las posibilidades de la vida, del sentir lo vivido. Y esto no puede darse más que en espacios ajenos a las lógicas institucionales. Es decir, en territorios flexibles que permitan el azar, la contingencia y los acontecimientos. Que permitan, justamente, que «algo» suceda, que «algo» acontezca, que «algo» se mueva. 


\section{QUEER O CRIP: DE LOS CUERPOS EXTRAÑOS A LOS CUERPOS ROTOS}

Si la pedagogía queer no se limita ni se centra exclusivamente en los temas ligados con la vivencia de las identidades GLBT sino que, fundamentalmente, busca la desestabilización del binomio normal/anormal, es pertinente plantearse su aplicación a otras realidades corporales, como puede ser el fenómeno de la discapacidad. Realidades hipercorporalizadas, reducidas a un cuerpo físico, que no se ajustan al estándar. Realidades medicalizadas y raptadas por un paradigma biomédico que ignora la confusión entre norma biológica y norma social. Realidades construidas socialmente que se han naturalizado permanentemente. Así pues, cabe preguntarse ¿Por qué necesitamos una pedagogía queer? Y ¿porque la necesitamos especialmente en materia de discapacidad? Es necesario hablar de pedagogía queer en el terreno de la discapacidad porque dicha teoría se ha preocupado de articular la cuestión de la alteridad en un registro de apropiación y subversión, cuestiones que, al fin y al cabo, son potencialmente posibilitadoras en materia de educación especial. En 1973 la homosexualidad era considerada un desorden psiquiátrico y actualmente la transexualidad todavía lo es. Ambos colectivos han vivido aislados y estigmatizados como anormales. Este paralelismo no significa que la cultura queer y la cultura de la discapacidad partan de la misma experiencia de opresión. Entre otras convergencias ambos colectivos han sido considerados como monstruosos y en ambos casos se ha puesto en juego la cuestión del orgullo. La cultura queer —si es que se puede hablar de cultura- celebra la diferencia, sin ocultarla, hasta la exhibición descarada. Entre otras cuestiones, esta cuestión del orgullo ocupa un lugar relevante en relación a lo que la teoría queer puede ofrecer al ámbito de la discapacidad.

Las personas con discapacidad que se autodenominan orgullosamente freaks fortalecen su sentido de resistencia, señalan una verdad y refuerzan su identidad. Pero Clare también se pregunta si en dicha autodenominación se puede estar olvidando una parte de la historia ligada a la segregación, la exclusión y el dolor (1999). Al respecto es evidente que las personas con discapacidad que se encuentran en momentos de negación, de tristeza o de rehabilitación no tienen la misma relación con la palabra freak. El proceso de asimilación y normalización no tiene nada que ver con su significado. La relación con este concepto será diferente para aquellas personas que ya tienen una historia ligada a la exposición y a la diferencia. Nos podemos preguntar: ¿dónde está el orgullo de una historia ligada a la exposición, corrección, segregación y eliminación? Para Clare las personas con discapacidad que se autodenominan freaks ayudan a construir una cultura de la discapacidad y ayudan también a trabajar para romper con la cultura dominante. Al utilizar la palabra freak se construye el orgullo de una herencia de siglos ubicada en los 
márgenes. La historia que durante tantos años ha colocado a las personas con discapacidad en el escenario del odio, la vergüenza y en ocasiones también en el escenario de la subversión y la resistencia exige la utilización de la palabra freak, no sólo como símbolo de orgullo sino también para dar testimonio de siglos de discriminación. La cuestión importante no es porque a unos les gusta mantener relaciones sexuales nada convencionales o que les pasa a los cuerpos de los otros, sino porque al resto les incomoda tanto su visión.

Es en estos términos donde cabe contextualizar lo que se ha dado en llamar Crip Theory, la cual no deja de ser una epistemología queer aplicada al campo de la discapacidad. Teoría maricona y teoría lisiada son dos maneras de comprender una diferencia inaceptable para el sistema. La obra de McRuer plantea la construcción de la normalidad corporal, la heterosexualidad y las conexiones entre ambas relacionando dicha «normalidad» con la historia y política económica de la visibilidad de la discapacidad y la homosexualidad (2006). El sistema de normalización corporal produce un determinado sentido sobre la discapacidad que está profundamente ligado al sistema heterosexista o de obligatoriedad heterosexual que produce rareza, extrañeza o queerness. De forma que para McRuer la heterosexualidad obligatoria es contingente a la obligatoriedad de la corporalidad «normal» y viceversa. En definitiva, la discapacidad, como la homosexualidad, son el fruto de una biopolítica y una anatomopolítica de las poblaciones y los cuerpos respectivamente.

La Crip Theory está relacionada con el estudio de la conceptualización y materialización de órganos y discapacidades en diferentes territorios culturales y como estos podrían ser comprendidos desde la resistencia a la homogeneización cultural. La Crip Theory también está relacionada con la autoidentificación crip en la calle. Es decir, con la destrucción de bordillos, con sillas de ruedas encadenadas a los autobuses u otras estaciones, reivindicando servicios comunitarios e instalaciones para la independencia de dichos sujetos. Los activistas con discapacidad que se han identificado como crip lo han hecho como respuesta a la norma que impone una «corporalidad-capaz» y como respuesta a la subordinación y opresión que, a consecuencia de este imperativo, sufren algunos cuerpos. Cuerpos estigmatizados por una cultura que no quiere o no puede adaptarse a la presencia de corporalidades diferentes. Los artistas crip tienen orgullo y presentan alternativas alrededor de la abyección. Es decir, en cierta manera juegan con la cuestión de la monstruosidad como provocación y posibilidad de existencia.

Es evidente que la educación que quiera inscribirse en este planteamiento debe alejarse de las cuestiones de la normalidad y la integración pero debe, especialmente, alejarse de la dicotomía ayudante/ayudado generando 
otros espacios de relación, otra lógica en la construcción del saber y, posiblemente, otros objetivos en la transmisión del saber. Es necesaria la construcción de territorios educativos y políticos que faciliten la apropiación de etiquetas, la invención de soluciones propias frente al malestar, que se alejen del deseo de integrar, que consideren seriamente el saber de los sujetos sobre aquello que «les pasa», que sacudan, que provoquen... Es necesaria una recuperación de la dimensión simbólica de los cuerpos como forma de resistencia a los modelos de producción biopolítica, lo cual, supone otra lógica en la articulación de servicios y recursos dirigidos a las personas con discapacidad. Supone otra epistemología de la discapacidad capaz de generar otras significaciones sobre la misma discapacidad que superen la normalización corporal.

I am not one of the physically challenged-

I'm a sock in the eye with a gnarled fist

I'm a French kiss with cleft tongue

I'm orthopedic shoes sewn on the last of your fears

I am not one of the differently abled-

I'm Eve I'm Kali

I'm The Mountain That Never Moves

I've been forever I'll be here forever

I'm the Gimp

I'm the Cripple

I'm the Crazy Lady

I'm the Woman With Juice.

Cheryl Marie Wade 


\section{NOTAS}

[1] Tal y como expone de forma detallada Carbonell: «El caso arranca el 3 de agosto de 1982, cuando un oficial de policía acude al domicilio particular de Michael Harwick en el barrio de Virginia Highlands a dejarle un aviso de arresto por no haber comparecido en un juicio que se le seguía por beber en público. Alguien de la casa le permitió la entrada al oficial de policía y le dijo que podía encontrar a Michael en el piso de arriba. El policía subió y abrió la puerta de un dormitorio, encontrando a Michael y a otro hombre practicando sexo oral. El oficial arrestó a ambos y los llevó a la estación de policía donde con términos muy gráficos les dijo a todos los presentes la razón por la que había arrestado a los dos hombres. El mismo policía era conocido por su aversión a los homosexuales y ya había detenido a varios más anteriormente. Los detenidos fueron puestos en libertad doce horas después de su arresto, pero un abogado de la American Civil Liberties Union (ACLU) se puso en contacto con Michael para ver si quería intentar impugnar la ley de Georgia que sancionaba la sodomía» (2009). 


\section{REFERENCIAS BIBLIOGRÁFICAS}

Berlant, L.; Warner, M. (2002). Sexo en público, en Mérida, R. (Ed.) Sexualidades transgresoras: Una antología de estudios queer. Barcelona: Icaria, 229-257.

Butler, J. (2001). El género en disputa. Mexico: Paidós.

Butler, J. (2002a). Cuerpos que importan. Sobre los límites materiales y discursivos del «sexo». Barcelona: Paidós.

Butler, J. (2006). Deshacer el género. Barcelona: Paidós.

Britzman, D. P. (1995). Is there a queer pedagogy? Or, stop reading straight, Educational Theory, 45, 151-165.

Britzman, D. P. (2002). La pedagogía transgresora y sus extrañas técnicas, en Mérida, R. M. (Ed.) Sexualidades transgresoras. Una antología de estudios queer. Barcelona: Icaria, 197-228.

Carbonell, M. (2009). Bowers versus Hardwick: cuando el derecho entra en la recámara. Disponible en http://www.miguelcarbonell.com/articulos/Bowers.sht $\mathrm{ml}$ [Consulta 2010, 9 de octubre]

Chase, C. (2005). Hermafroditas con actitud: cartografiando la emergencia del activismo político intersexual, en Grupo de Trabajo Queer (eds) El eje del mal es heterosexual: Figuraciones, movimientos y prácticas feministas queer. Madrid: Traficantes de sueños, 87-111.

Clare, E. (1999). Exile \& Pride: disability, queerness and liberation. U.S.A.: South End Press.

Córdoba, D; Sáez, J; Vidarte, P. (2005). Teoría queer: politicas bolleras, maricas, trans, mestizas. Barcelona: Egales.

Curran, G. (1998). Challenging heterosexuality and homophobia in schools. [En línea] Disponible en: www.aare.edu.au/index.htm. [consulta 2010, 23 de junio].
Curan, G. (2002). Young Queers Getting Together: Moving Beyond Isolation and Loneliness. Ph.D. Faculty of Education, University of Melbourne.

Ferrús, B. (2007). ¿Por qué necesitamos una pedagogía queer? La Vanguardia, 5 de setiembre.

Gamson, J. (2002). ¿Deben autodestruirse los movimientos identitarios? Un extraño dilema, en Mérida, R.M. (Ed) Sexualidades transgresoras: una antología de estudios queer. Barcelona: Icaria, 141-172.

Gough, A. \& Gough, N. (2002). Queer(y)ing Environmental Education Research: A Discursive In(ter)vention, Proceedings $10^{\text {th }}$ Contemporary Approaches to Research in Mathematics, Science and Environmental Education Symposium. Deakin University, 9-10 de diciembre 2002.

Gore, J. (2000). Disciplinar los cuerpos: sobre la continuidad de las relaciones de poder en pedagogía, en Popkewitz, T.; Brennan, C. (coords) El desafío de Foucault: discurso, conocimiento y poder en la educación. Barcelona: Pomares-Corredor, 228-249.

Grace, A. P. (2001). Being, Becoming, and Belonging as a Queer Citizen Educator: The Places of Queer Autobiography, Queer Culture as Community, and Fugitive Knowledge, Proceedings National Conference CASAE-ACÉÉA.

Grupo de Trabajo Queer (2005). El eje del mal es heterosexual: figuraciones, movimientos y prácticas feministas queer. Madrid: Traficantes de sueños.

Hénaff, M. (1980). Sade. La invención del cuerpo libertino. Barcelona: Destino.

Jennings, K. (1994). One Teacher in Ten: Gay and Lesbian Educators Tell Their Stories. Boston: Alyson Publications. 
Kopelson, K. (2002). Dis/Integrating the Gay/Queer Binary: Reconstructed Identity Politics for a Performative Pedagogy, College English, 65 (1), 17-35.

Khayatt, M. D. (1992). Lesbian Teachers: An Invisible Presence. Albany: University of New York Press.

Lizarraga, X. (2003). Una historia sociocultural de la homosexualidad. Barcelona: Paidós.

Lorde, A. (1984). La hermana y la extranjera. Madrid: Horas.

Lyotard, J.-E. (1999). La diferencia. Barcelona: Gedisa

Llamas, R. (1998). Teoría torcida: Prejuicios y discursos en torno a "la homosexualidad». Madrid: Siglo Veintiuno.

Mèlich, J. C. (2010). Ética de la compasión. Barcelona: Herder.

Mérida, R. (2002). (Ed.) Sexualidades transgresoras. Una antología de estudios queer. Barcelona: Icaria.

Morris, M. (2005). El pie zurdo de Dante pone en marcha la teoría queer, en Talburt, S.; Steinberg, S. (Eds.) Pensando queer: Sexualidad, cultura y educación. Barcelona: Graó, 35-55.

McKeehen, L. (2002). Critical Performative Pedagogy: Augusto Boal's Theatre of the Oppressed in the English as a Second Language Classroom. Lousiana State University: Ph. D.

McLaren, P. (1997). Pedagogía crítica y cultura depredadora: políticas de oposición en la era posmoderna. Barcelona: Paidós.

McRuer, R. (2006). Crip Theory: Cultural Signs of Queerness and Disability. New York: New York University Press.

Peter, (2000). A People's History of The . Nueva York: Penguin Books.
Pie, A. (2009). Educació social i Teoria Queer: de l'alteritat o les dissidències pedagògiques. Barcelona: UOC.

Pinar, W. F. (1998). Queer Theory in Education. Mahwah: Erlbaum.

Planella, J. (2006). Cuerpo, cultura y educación. Bilbao: Desclée de Brower.

Planella, J. (2007). Los monstruos. Barcelona: Ediuoc.

Popkewitz, T. S.; Brennan, M. (Comp.) (2000). El desafío de Foucault: discurso, conocimiento y poder en la educación. Barcelona: Pomares-Corredor.

Sedgwick, E. K. (1998). Epistemología del armario. Barcelona: La tempestad.

Sedgwick, E. K (2002). A (queer) y ahora, R. Mérida (Ed.) Sexualidades transgresoras. Una antología de estudios queer Barcelona: Icaria, 29-54.

Spivak, G. Ch. (1992). Acting Bits/Identity Talk. Critical Inquiry, 18 (4), 770-803.

Talburt, S.; Steinberg, S. R (2005). Pensando queer: Sexualidad, cultura y educación. Barcelona: Graó.

Weems, L. (1999). Pestalozzi Perversity, and the Pedagogy of Love, in Letts, W. J. \& Sears, J. T. (Eds.) Queering Elementary Education: Advancing the Dialogue about Sexualities and Schooling. Maryland: Rowman and Littlefield, 27-36.

Woog, D. (1995). School's Out: The Impact Of Gay And Lesbian Issues On America's Schools. Los Ángeles: Alyson Publications.

Uunks, G. (1995). (ed.) The Gay Teen: Educational Practice and Theory for Lesbian, Gay and Bisexual Adolescents. Nova York: Routledge. 


\section{PALABRAS CLAVE}

Ciencias de la Educación, Cuestiones de Género, Política de la Educación, Orientación Sexual, Actitudes Sociales, Homosexualidad

\section{KEYWOORDS}

Science Education, Gender Issues, Politics of Education, Sexual Orientation, Social Attitudes, Homosexuality.

\section{PERFIL ACADÉMICO Y PROFESIONAL DE LOS AUTORES}

Jordi Planella Ribera, Doctor en Pedagogía por la Universidad de Barcelona. Profesor de la Universitat Oberta de Catalunya (UOC). Está acreditado como Catedrático del área de Teoría e Historia de la Educación. Ha sido el investigador principal del proyecto Controversias tecnocientíficas y participación ciudadana en las políticas sociales de dependencia (CSO20806308-C02-02). Su línea de investigación se centra en la Pedagogía de la Diversidad Funcional y el Pensamiento Pedagógico Contemporáneo.

Asun Pie Balaguer, Doctora en Pedagogía por la Universidad de Barcelona. Profesora de los Estudios de Psicología y Ciencias de la Educación de la Universitat Oberta de Catalunya (Barcelona). Participa en el proyecto de investigación Controversias tecnocientíficas y participación ciudadana en las politicas sociales de dependencia (CSO208-06308-C02-02). Su línea de investigación se centra en la Pedagogía Queer y la Discapacidad.

Dirección de los autores: Jordi Planella Ribera y Asun Pie Balaguer

Facultad de Psicología y Ciencias de la

Educación

Universitat Oberta de Catalunya

Rambla del Poblenou, 156

08018 BARCELONA

E-mail: apieb@uoc.edu

E-mail: jplanella@uoc.edu

Fecha Recepción del Artículo: 08. Julio. 2010

Fecha Revisión del Artículo: 15. Octubre. 2010

Fecha Aceptación del Artículo: 16. Diciembre. 2010

Fecha de Revisión para publicación: 14. Junio. 2011 
